\title{
Effets des éclusées hydroélectriques en rivière : diagnostic hydroécologique et aide à la gestion. Exemple de la Fontaulière (Ardèche)
}

\author{
par Sylvie Valentin \\ CEMAGREF, Division Biologie des Ecosystèmes Aquatiques, Laboratoire d'Hydroécologie Quantitative, \\ 3 bis, quai Chauveau, CP 220, 69336 Lyon Cedex 09
}

\section{INTRODUCTION}

L'étude des réponses des organismes vivants aux variations des facteurs environnementaux constitue un axe fondamental de la recherche en écologie et permet de mieux comprendre la répartition des peuplements et des individus dans l'espace et dans le temps. En rivière, le débit constitue le principal facteur abiotique organisant l'écosystème lotique. Hynes (1970) évoquait déjà l'importance de la variabilité hydrologique comme facteur structurant les peuplements lotiques. L'influence de ce facteur s'exerce depuis l'échelle des processus géomorphologiques jusqu'à une échelle locale sur les caractéristiques hydrodynamiques de l'habitat (Newbury, 1984). Statzner et Higler (1986) attribuent aux caractéristiques hydrauliques un rôle prépondérant dans la répartition spatiale des communautés benthiques; les variations du débit constitueraient le principal facteur abiotique expliquant la distribution et l'abondance des communautés aquatiques (Statzner et al., 1988; Power et al., 1988; Poff et Ward, 1989). Les fluctuations saisonnières naturelles du régime hydrologique jouent donc un rôle majeur dans la structura- tion de l'écosystème et, par conséquent, les modifications artificielles du débit peuvent avoir des effets non négligeables.

De nombreux aménagements ont été construits pour répondre à des objectifs variés selon les différents usages de l'eau. Parmi ceux-ci, la production d'électricité s'accompagne d'installations plus ou moins importantes dont l'exploitation entrainne souvent une modification artificielle des régimes hydrologiques en aval. Certaines usines hydroélectriques peuvent fonctionner de manière intermittente pour répondre aux demandes d'énergie à certains moments de la journée. Ce mode de gestion entraîne des lâchers d'eau appelés éclusées hydroélectriques, se traduisant par des variations de débit généralement importantes, brutales et fréquentes (jusqu'à trois fois par jour). L'écosystème aquatique est donc soumis à des modifications particulières des caractéristiques hydrodynamiques de l'écoulement (hauteurs d'eau, vitesses du courant), provoquant des modifications de l'habitat physique des organismes présents (poissons, invertébrés, algues).

Les fluctuations des paramètres hydrodynamiques en situation naturelle sont souvent moins brutales et moins fré-

Hydropower generation induces artificial fluctuations of flow and thus of hydrodynamic parameters in rivers, downstream of hydroelectric impoundments. On the Fontaulière river (Ardèche basin, France), the ecological effects of two different hydropeaking regimes were tested and compared to a reference upstream reach. Fish. invertebrate and epilithon communities were surveyed in these different reaches. The results enabled to classify the factors responsible for the observed effects. The base flow between peaks was the most important for the studied site. When it was too low, aquatic communities were destructured in comparison with the natural reach. These results enabled to suggest a current velocity threshold to respect in order to determine acceptable base flow for this type of stream. They also enabled to guide for the impact evaluation of hydropeaking sites. An ecological diagnosis should include the study of the structure and composition of the communities. 
quentes car elles ont lieu à un tout autre pas de temps. De très fortes crues peuvent cependant entraîner des déplacements d'organismes vivants vers l'aval, voire même de fortes mortalités selon le degré de remaniement du substrat, les refuges disponibles et les capacités des organismes à les utiliser (Sedell et al., 1990 ; Detenbeck et al., 1992 ; Lancaster et Hildrew, 1993; Scarsbrook et Townsend, 1993). Dans ce cas, il existe des mécanismes permettant aux organismes de se maintenir dans le milieu et de répondre à différents niveaux de variation.

Nous proposons ici d'examiner le cas de la Fontaulière, cours d'eau situé en Ardèche. Ce site est soumis à un régime d'éclusées, avec deux niveaux de débit plancher dans deux secteurs distincts. L'effet d'une crue centennale a par ailleurs été suivi sur ce site. A partir de l'étude des peuplements de poissons, nous discuterons de l'impact des deux régimes d'éclusées par rapport à celui de la crue. L'étude des réponses des peuplements d'invertébrés réalisée sur le même site (Valentin et al., 1995) fournit des renseignements complémentaires pour situer les différents types de variations du débit en terme de perturbation. A l'aide de ces résultats, nous apporterons des éléments d'aide à la gestion et au diagnostic.

\section{II 賸 MATÉRIELS ET MÉTHODES}

\subsection{Site d'étude}

La Fontaulière est un affluent de l'Ardèche à l'amont d'Aubenas. La rivière parcourt 20 kilomètres pour une superficie du bassin versant de $134 \mathrm{~km}^{2}$, entre 1100 et 300 mètres d'altitude, sur des roches d'origine basaltique. La qualité de l'eau est bonne (classe 1A, SRAE Rhône Alpes, 1991).

Le régime hydrologique est de type cévenol, c'est-à-dire pluvial avec une influence méditerranéenne donnant lieu à des crues parfois brutales, et un étiage sévère en été

Dans le secteur naturel situé en amont du principal affluent de la Fontaulière (la Bourges), le module interannuel est environ de $2,5 \mathrm{~m}^{3} \cdot \mathrm{s}^{-1}$ (fig. 1 ), avec un débit moyen mensuel en étiage d'été égal à $0,2 \mathrm{~m}^{3} \cdot \mathrm{s}^{-1}$ en moyenne. Ce module passe à $4,7 \mathrm{~m}^{3} \cdot \mathrm{s}^{-1}$ en aval de la confluence avec la Bourges, avec un débit d'étiage (moyenne des débits moyens mensuels des mois les plus secs) de $0,4 \mathrm{~m}^{3} \cdot \mathrm{s}^{-1}$. La retenue de Pont de Veyrières, située à environ un kilomètre en aval (fig. I), reçoit, en plus des apports naturels de la rivière, les eaux turbinées par éclusées à l'usine de Montpezat, en provenance du bassin de la Loire (lac d'Issarlès, retenues du Gage et de la Palisse). Ces apports représentent un débit moyen annuel de $7 \mathrm{~m}^{3} \cdot \mathrm{s}^{-1}$, et se traduisent, en aval du barrage de Pont de Veyrières, par des éclusées de $19 \mathrm{~m}^{3} . \mathrm{s}^{-1}$. Le débit plancher restitué entre les éclusées en aval du barrage est de $1 \mathrm{~m}^{3} \cdot \mathrm{s}^{-1}$, soit environ $1 / 9^{c}$ du module transitant dans ce secteur: Le rapport entre débit d'éclusée et débit plancher est proche de 15 .

Une microcentrale dérive une partie de ce débit sur un kilomètre (fig. l), avec un débit minimum fixé dans la rivière à $1 / 40^{\mathrm{e}}$ du module naturel amont, soit $0,12 \mathrm{~m}^{3} \cdot \mathrm{s}^{-1}$, correspondant à $1 / 100^{\mathrm{e}}$ du module transitant effectivement dans le secteur. Les éclusées sont alors variables de 12 à 19 $\mathrm{m}^{3} \cdot \mathrm{s}^{-1}$ selon le fonctionnement de la microcentrale (débit d'équipement de $7 \mathrm{~m}^{3} \cdot \mathrm{s}^{-1}$ ). Le rapport entre débit maximum d'éclusée et débit plancher est supérieur à 150 .

Durant la période d'étude (1991 à 1993), une crue centennale a eu lieu le 22 septembre 1992, après 24 heures de pluie d'orage en continu, le débit a augmenté très rapidement, pour atteindre en moins d'une heure un maximum estimé à $1000 \mathrm{~m}^{3} \cdot \mathrm{s}^{-1}$. Cette crue a détruit le canal de dérivation de la microcentrale du Pradel. Le régime hydraulique en aval du seuil du Pradel est donc devenu identique au régime restitué en aval de Pont de Veyrières, avec le même débit plancher $\left(1,35 \mathrm{~m}^{3} \cdot \mathrm{s}^{-1}\right)$ et les mêmes débits d'éclusée $\left(19 \mathrm{~m}^{3} \cdot \mathrm{s}^{-1}\right)$. De gros blocs (certains mesurant plus de $1,5 \mathrm{~m}$ de diamètre) ont été déposés sur les berges, dans les prés bordant la rivière, témoignant d'un remaniement important du substrat.

\subsection{Stations d'étude (fig. 1 et tabl. I)}

Les stations ont été déterminées après une description des faciès d'écoulement selon Malavoi (1989), dans les différents secteurs (un secteur naturel, deux secteurs influencés). Elles doivent d'une part représenter au mieux la diversité morphologique de chaque secteur, et d'autre part, contenir une succession de faciès variés nécessaires aux différentes espèces présentes (poissons et invertébrés) à chaque stade de leur développement.

Du fait de la courte distance entre la Bourges et la retenue de Pont de Veyrières, la station I (S1) a été choisie en amont de la confluence avec la Bourges. Deux stations ont été choisies dans le secteur soumis à éclusées avec des régimes hydrauliques différents : la station 2 (S2) est à 800 mètres en aval du barrage de Pont de Veyrières, la station 3 (S3) est dans le secteur court-circuité par la microcentrale du Pradel.

\subsection{Echantillonnages des peuplements de poissons}

Les peuplements de poissons ont été échantillonnés dans chaque station par pêche électrique (courant continu de 400

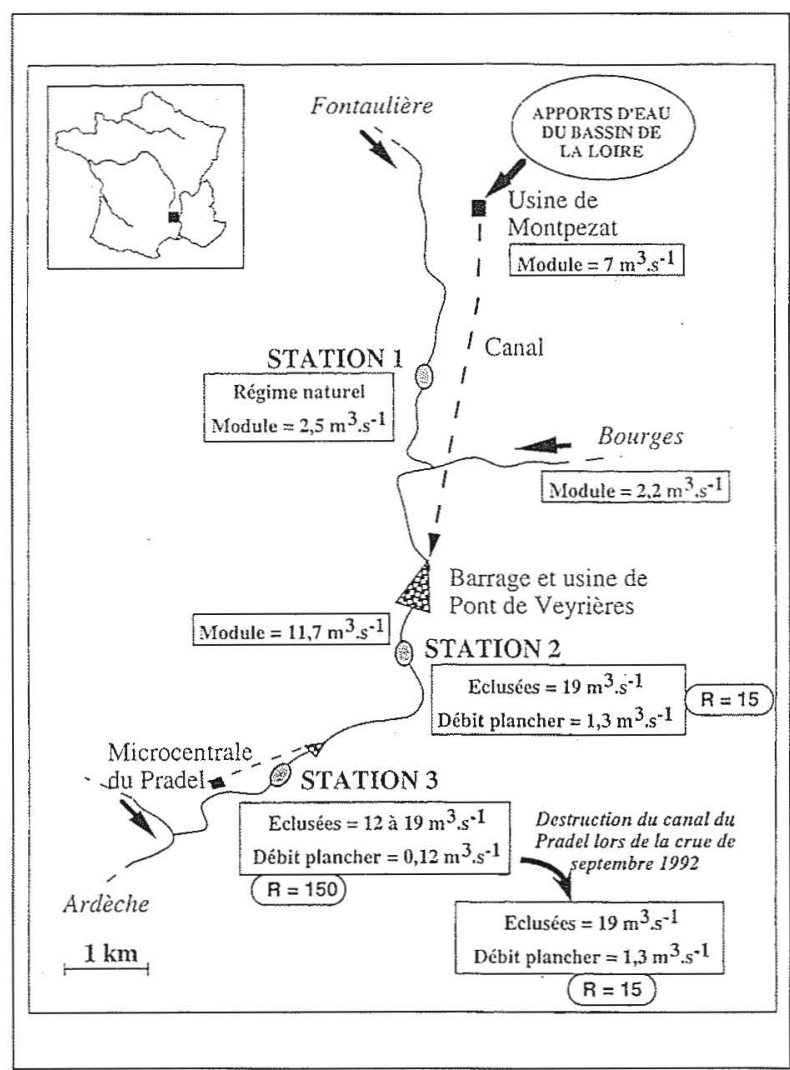

1. Schéma de la Fontaulière et emplacement des stations d'étude. ' $R$ ' correspond au rapport entre la valeur $d u$ débit maximum d'éclusées et la valeur du débit plancher. 
Tableau 1. - Caractéristiques des trois stations d'étude (S1, S2 et S3) de la Fontaulière.

\begin{tabular}{|c|c|c|c|}
\hline \multirow{2}{*}{ Caractéristiques } & \multicolumn{3}{|c|}{ Stations } \\
\hline & S1 & $\mathrm{S} 2$ & S3 \\
\hline Régime hydraulique $\left(\mathrm{m}^{3} \cdot \mathrm{s}^{-1}\right)$ & naturel & $\begin{array}{c}\text { débit de base - } \\
\text { éclusées } \\
(1,35-19)\end{array}$ & $\begin{array}{c}\text { débit de base } \\
\text { éclusées } \\
\left(0,12^{*}-19\right)\end{array}$ \\
\hline Module $\left(\mathrm{m}^{3} \cdot \mathrm{s}^{-1}\right)$ & 2,5 & 11,7 & 11,7 \\
\hline Surface du bassin versant $\left(\mathrm{km}^{2}\right)$ & 55 & 125 & 128 \\
\hline Distance à la source $(\mathrm{km})$ & 12 & 16 & 18 \\
\hline Altitude $(\mathrm{m})$ & 400 & 350 & 325 \\
\hline Pente $(\%)$ & 6,4 & 7,3 & 3,1 \\
\hline Largeur moyenne $(\mathrm{m})$ & 7 & 16 & 12 \\
\hline Longueur (m) & 89 & 123 & 83 \\
\hline Faciès ( $\%$ de la longueur): & & & \\
\hline plat & 27 & 23 & 43 \\
\hline radier-rapide & 44 & 77 & 26 \\
\hline mouille & 29 & $24 * *$ & 31 \\
\hline
\end{tabular}

(*) Avant la crue de septembre 1992.

(*a) Mouille latérale, parallèle à un radier.

à 600 volts et 1 à 2 ampères délivré par un matériel homologué de type «Héron»). Compte tenu de la largeur des stations, deux anodes ont été utilisées en parallèle. Deux campagnes de pêche ont pu être réalisées avant la crue $(\mathrm{Cl}$ et $\mathrm{C} 2$, tabl. $I I)$. Lors de la première campagne $(\mathrm{Cl})$, la station 2 n'a pas pu être pêchée en raison d'un débit lâché à Pont de Veyrières trop élevé (soutien d'étiage). Deux campagnes (C3 et C4) ont été effectuées dans l'année suivant cette crue (respectivement à six mois et un an de la crue).

A chaque campagne, les poissons ont été pêchés en deux passages successifs séparés par un délai d'au moins une heure. Tous les poissons pêchés ont été identifiés à l'espèce, mesurés (longueur totale) et pesés, puis remis à l'eau après le deuxième passage. Les effectifs et les biomasses par station ont été estimés pour chaque espèce à partir des résultats des deux passages selon la méthode de Carle et Strub (1978), méthode expliquée et validée par Cowx (1983) et Gerdeaux (1987).

\subsection{Echantillonnages des peuplements d'invertébrés}

Des prélèvements d'invertébrés ont été parallèlement réalisés au filet de type Surber sur les trois stations de la Fontaulière lors de cinq campignes. Deux campagnes ont été réalisées avant la crue. Deux cumpagnes ont ensuite été effectuées trois semaines et trois mois après la crue, sur la station de référence uniquement (S1), car le débit était trop élevé pour échantillonner aussi le.. stations 2 et 3 . La cinquième campagne a été réalisée sur les trois stations six mois après la crue. Le protocole détaillé ainsi que les résultats sont exposés par Valentin et al. (1995). Les résultats seront repris ici pour compléter l'analyse des réponses biologiques aux différentes modifications du débit.

Tableau II. - Dates des campagnes (Ci) de pêches électriques effectuées sur chaque station de la Fontaulière. (\#) campagnes effectuées avant la crue du 22 septembre 1992.

\begin{tabular}{|clll|}
\hline \multirow{3}{*}{ Campagne } & \multicolumn{3}{c|}{ Stations } \\
\cline { 2 - 4 } & S1 & S2 & S3 \\
\hline C1 \# & Août-91 & - & Août-91 \\
C2 \# & Mai-92 & Mai-92 & Mai-92 \\
C3 & Avril-93 & Avril-93 & Avril-93 \\
C4 & Sept-93 & Sept-93 & Sept-93 \\
\hline
\end{tabular}

\section{RÉSULTATS}

\subsection{Poissons avant la crue}

Les résultats des pêches réunis dans le tablecal $/ / I$ montrent des différences très nettes entre les stations (fig. 2). Le peuplement de la station de référence (S1) est composé uniquement de truite et de vairon. Le peuplement de la station 2 est également dominé par la truite et le vairon, mais caractérisé par plusieurs autres espèces en très faible densité : chabot, goujon, loche, blageon, anguille. Le peuplement de la station 3 est dominé par le vairon, la loche et le goujon. Le blageon est également assez abondant, surtout lors des deux premières campagnes. Des chevaines, anguilles et chabots ont été également été capturés lors de certaines campagnes, en très faible densité.

Les densités et biomasses de truite par hectare sont plus faibles dans la station 2 que dans la station 1. Dans la station 3 , les truites sont moins abondantes que dans la station 2, et nettement moins abondantes que dans la station 1 .

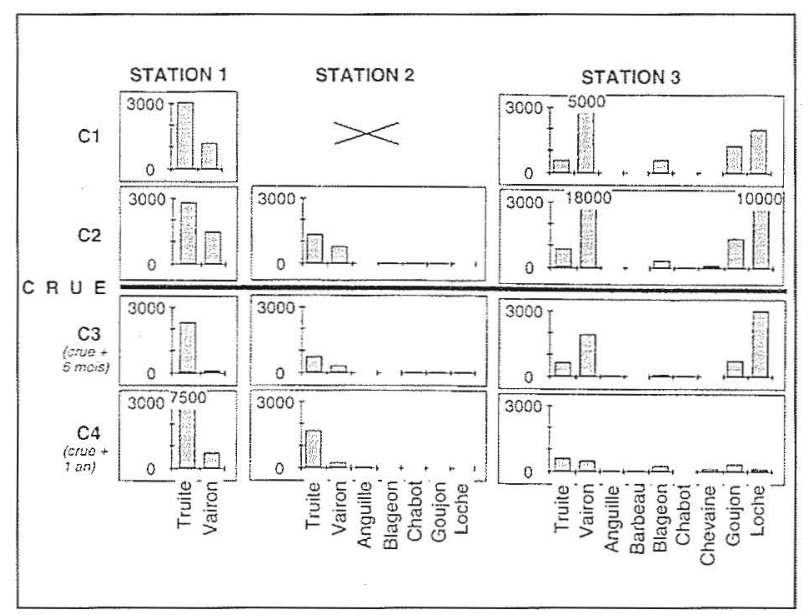

2. Densités (ind/ha) des différentes espèces de poissons, estimées par la méthode de Carle et Strub (1978), lors des 4 campagnes de pêche ( $\mathrm{Cl}$ à $\mathrm{C} 4$, en ligne) réalisées sur les trois stations de la Fontaulière (stations 1 à 3 , en colonne). 
Tableau III. - Résultats des pêches électriques réalisées sur la Fontaulière lors de chacune des quatre campagnes (C1 à $\mathrm{C} 4$ ). Les effectifs $(N)$ et biomasses (B, en grammes) ont été estimés par la méthode de Carle et Strub (1978) et ont été ramenés à 100 mètres de linéaire pour chaque station ( 1 1, S2 et $\mathrm{S} 3$ ). IC représente l'intervalle de confiance de l'effectif à $95 \%$ et $\mathrm{p}^{\text {' }}$ est la probabilité de capture.

\begin{tabular}{|c|c|c|c|c|c|c|c|c|c|c|c|}
\hline & & & & & & Espè & de pois & & & & \\
\hline & & & ANG & BAF & BLN & $\mathrm{CHA}$ & $\mathrm{CHE}$ & GOU & LOF & TRF & VAI \\
\hline & S1 & & - & - & - & - & - & - & - & 213 & 83 \\
\hline & & B & - & - & - & - & - & - & - & 11591 & 49 \\
\hline & & & - & - & - & - & - & - & - & 0,51 & 0,51 \\
\hline C1 & & & - & - & - & - & - & - & - & 48 & 26 \\
\hline & S3 & $N$ & - & - & 68 & - & - & 146 & 235 & 68 & 595 \\
\hline & & B & - & - & 51 & - & - & 2575 & 345 & 3519 & 774 \\
\hline & & $p$ & - & - & 0,76 & - & - & 0,26 & 0,62 & 0,83 & 0,54 \\
\hline & & ic & - & - & 6 & - & - & 117 & 29 & 2 & 72 \\
\hline & S1 & $N$ & - & - & - & - & - & - & - & 194 & 102 \\
\hline & & B & - & - & - & - & - & - & - & 4628 & 186 \\
\hline & & $p$ & - & - & - & - & - & - & - & 0,78 & 0,73 \\
\hline & & ic & - & - & - & - & - & - & - & 10 & 9 \\
\hline $\mathrm{C} 2$ & $\mathrm{~S} 2$ & $N$ & - & - & 1 & 3 & - & 3 & - & 241 & 137 \\
\hline & & $B$ & - & - & 2 & 20 & - & 35 & - & 9009 & 376 \\
\hline & & $p$ & - & - & 1,00 & 1,00 & - & 0,44 & - & 0,63 & 0,51 \\
\hline & & IC & - & - & & & - & 2 & - & 19 & 27 \\
\hline & S3 & $\mathrm{N}$ & - & - & 40 & 1 & 12 & 162 & 1220 & 103 & 2174 \\
\hline & & B & - & - & 655 & 10 & 3258 & 3019 & 2312 & 5778 & 4045 \\
\hline & & $\mathrm{p}$ & - & - & 0,49 & 1,00 & 0,50 & 0,62 & 0,22 & 0,67 & 0,35 \\
\hline & & IC & - & - & 17 & & 6 & 24 & 650 & 14 & 405 \\
\hline & $\mathrm{S} 1$ & $N$ & - & - & - & - & - & - & - & 227 & 11 \\
\hline & & B & - & - & - & - & - & - & - & 4029 & 42 \\
\hline & & $\mathrm{p}$ & - & - & - & - & - & - & - & 0,67 & 0,81 \\
\hline & & IC & - & - & - & - & - & - & - & 18 & 0 \\
\hline C3 & S2 & $N$ & - & - & - & 1 & - & 3 & 1 & 100 & 47 \\
\hline & & B & - & - & - & 24 & - & 61 & 1 & 3703 & 59 \\
\hline & & $p$ & - & - & - & 0,67 & - & 1,00 & 1,00 & 0,59 & 0,91 \\
\hline & & ic & - & - & - & 0 & - & & & 16 & 0 \\
\hline & S3 & $N$ & 1 & - & 8 & 1 & - & 88 & 361 & 77 & 231 \\
\hline & & B & 220 & - & 68 & 24 & - & 1653 & 941 & 1446 & 467 \\
\hline & & $\mathrm{p}$ & 1,00 & - & 1,00 & 0,50 & - & 0,47 & 0,08 & 0,64 & 0,83 \\
\hline & & ic & & - & & 0 & - & 34 & 466 & 15 & 7 \\
\hline & S1 & $N$ & - & - & - & - & - & - & - & 748 & 68 \\
\hline & & $B$ & - & - & - & - & - & - & - & 10072 & 85 \\
\hline & & $p$ & - & - & - & - & - & - & - & 0,53 & 0,82 \\
\hline & & ic & - & - & - & - & - & - & - & 70 & 3 \\
\hline $\mathrm{C}_{4}$ & $\overline{\mathrm{S} 2}$ & $N$ & 1 & - & - & - & - & - & - & 234 & 32 \\
\hline & & B & 121 & - & - & - & - & - & - & 10199 & 89 \\
\hline & & $\mathrm{p}$ & 1,00 & - & - & - & - & - & - & 0,50 & 0,18 \\
\hline & & ic & & - & - & - & - & - & - & 39 & 36 \\
\hline & S3 & $N$ & 2 & 1 & 30 & - & 13 & 40 & 14 & 74 & 59 \\
\hline & & $B$ & 333 & 902 & 1391 & - & 2499 & 1117 & 98 & 2426 & 223 \\
\hline & & $\mathrm{p}$ & 1,00 & 1,00 & 0,49 & - & 0,92 & 0,68 & 0,65 & 0,70 & 0,47 \\
\hline & & IC & & & 14 & - & 0 & 7 & 4 & 10 & 25 \\
\hline
\end{tabular}

Légende des codes pour les espèces de poissons du tableau ci-dessus.

\begin{tabular}{lll} 
Code & Nom vernaculaire & \multicolumn{1}{c}{ Nom latin } \\
& & \\
ANG & Anguille & Anguilla anguilla (L., 1758). \\
BAF & Barbeau tluviatile & Barbus barbus (L.. 1758). \\
BLN & Blageon & Leuciscus (telestes) souffia (Risso, 1826). \\
CHA & Chabot & Cottus gobio (L., 1758).
\end{tabular}

\subsection{Poissons après la crue}

Lors de la campagne C3 (six mois après la crue), on retrouve les mêmes espèces dans chacune des stations. On observe par contre des réductions de densités et biomasses pour toutes les espèces, les réductions étant moins importantes dans le cas de la truite. Les individus de cette espèce

$\begin{array}{ll}\text { Code } & \text { Nom vernaculaire } \\ \text { CHE } & \text { Chevaine } \\ \text { GOU } & \text { Goujon } \\ \text { LOF } & \text { Loche franche } \\ \text { TRF } & \text { Truite fario } \\ \text { VAI } & \text { Vairon }\end{array}$

Nom latin

Lenciscus cephalus (L., 1758) Gobio gobio (L., 1758). Noemacheilus barbatulus (L., 1758). Salmo trutta fario (L., 1758). Phoxinus phoxinus (L., 1758) sont essentiellement des $0+$ (jusqu'à $65 \%$ ) introduits par alevinage après la crue et avant la campagne $\mathrm{C} 3$.

Lors de la campagne $\mathrm{C} 4$ (un an après la crue), les densités et biomasses des différentes espèces sont nettement réduites sur la station 3 par rapport à la campagne C3. A l'inverse, sur les stations 1 et 2, les densités et biomasses ont augmenté pour la plupart. 


\subsection{Invertébrés : effet de la crue sur la station 1}

La diversité des peuplements d'invertébrés est rappelée au tableau $I V$, extrait des travaux de Valentin et al. (1995). On constate que lors de la troisième campagne (trois semaines après la crue), la diversité est beaucoup plus faible (tabl. IV). La densité globale a été réduite de $87 \%$ et la biomasse de $95 \%$ par rapport aux moyennes des deux campagnes ayant précédé la crue (d'après les résultats de Valentin et al., 1995). Lors de la cinquième campagne (six mois après la crue), le peuplement de la station 1 semble de nouveau comparable à celui des deux premières campagnes (tabl.IV); on peut donc considérer que l'effet de la crue est estompé (Valentin et al., 1995). Sur les trois stations, on s'autorisera alors à analyser conjointement les deux premières campagnes et la cinquième.

\subsection{Invertébrés : comparaison des trois stations}

La diversité des peuplements des stations 2 et 3 est inférieure à celle de la station 1 (tabl. IV). Valentin et al. (1995) montrent que les stations 2 et 3 sont dominées par un ou deux taxons (généralement des chironomes) et que des représentants de Plécoptères, Ephéméroptères et Diptères, présents dans la station 1 , sont absents des stations 2 et 3 .

Dans la station 3, avant l'augmentation du débit plancher (deux premières campagnes), la diversité est cependant un peu plus élevée. Elle est liée à l'apparition de taxons de milieu stagnant dans les faciès lents, totalement différents des taxons lénitophiles prélevés dans la station de référence. Cette discordance s'accompagne d'une surabondance algale excessive, avec des biomasses de chlorophylle-a dépassant des seuils de nuisance cités dans la littérature (Horner et al., 1983 ; Welch et al., 1988). Après l'augmentation du débit plancher sur la station 3, ces taxons de milieu stagnant disparaissent, et le peuplement de la station 3 ressemble davantage à celui de la station 2 (diversité plus faible qu'en station 1, peuplement moins discordant, d'après Valentin et al., 1995).

Valentin et al. (1995) ont relié les effets observés aux vitesses de courant dans les faciès les plus lents. En situation de débit plancher faible (discordances marquées), les vitesses de courant dans les faciès lents étaient de $1 \mathrm{~cm} . \mathrm{s}^{-1}$ en moyenne, avec des valeurs locales n'excédant pas $4 \mathrm{~cm} \cdot \mathrm{s}^{-1}$. A la station 2 et à la station 3 après relèvement du débit (effets moins déstructurants), ces vitesses étaient respectivement de $8 \mathrm{~cm} \cdot \mathrm{s}^{-1}$ et $30 \mathrm{~cm} \cdot \mathrm{s}^{-1}$.

Tableau IV. - Diversité (en terme de nombre de taxons et indice de Shannon) des peuplements d'invertébrés benthiques prélevés lors de cinq campagnes (extrait de Valentin et al., 1995).

(\#) campagnes effectuées avant la crue du 22 septembre 1992.

\begin{tabular}{|clcc|}
\hline Station & Date & $\begin{array}{c}\text { Nombre } \\
\text { de taxons }\end{array}$ & $\begin{array}{c}\text { Indice } \\
\text { de Shannon }\end{array}$ \\
\hline \multirow{3}{*}{ S1 } & fév. 92 \# & 66 & 4,3 \\
& mai 92 \# & 61 & 4,4 \\
& oct. 92 & 29 & 2,9 \\
& déc. 92 & 54 & 3,3 \\
& mars 93 & 56 & 3,1 \\
S2 & fév. 92 \# & 46 & 3,5 \\
& mai 92 \# & 37 & 1,5 \\
& mars 93 & 35 & 1,5 \\
S3 & fév. 92 \# & 56 & 2,6 \\
& mai 92 \# & 55 & 3,4 \\
& mars 93 & 42 & 2,6 \\
\hline
\end{tabular}

\section{IV 圈 DISCUSSION}

\subsection{Influence du régime d'éclusées sur les poissons (campagnes précédant la crue)}

Le peuplement piscicole de la station 3 (régime d'éclusées avec débit plancher faible) est nettement différent de celui des deux autres stations, avec moins de truites et une forte abondance d'autres espèces. Les espèces les plus abondantes sont discordantes par rapport à la station 1 de référence et par rapport à la station 2 (goujon, chevaine, vairon, loche). Leur abondance est à relier au débit plancher très faible transitant dans la station 3, imposant des conditions très lentiques entre les éclusées. L'abondance de la loche est directement liée à la source de nourriture (Horton, 1994); cette espèce se développe en effet dans des secteurs où le développement algal important favorise le développement des chironomes dont elle se nourrit principalement (Gaudin, comm. pers.). Le peuplement de la station 3 apparaît donc nettement influencé par la faible valeur du débit de base, bien que celui-ci soit interrompu par des débits d'éclusées.

Sur la station 2, le régime d'éclusées avec un débit de base plus soutenu semble se traduire surtout par une réduction relative des populations de truite (densités et biomasses à l'hectare), sans changement fondamental dans la composition spécifique par rapport à la situation de référence.

Les effets du régime d'éclusées sur le peuplement de poissons se manifestent donc à deux niveaux. Le facteur le plus important mis en évidence ici est la valeur du débit plancher.

\subsection{Effet de la crue sur les poissons}

La crue centennale a entrainé des diminutions sensibles des densités et biomasses pour toutes les espèces, mais la composition spécifique n'a pas été modifiée. L'évolution des densités de truite n'a pas pu être correctement analysée puisque des alevinages ont artificiellement augmenté ces densités.

Les fortes réductions du peuplement observées sur la station 3 lors de la quatrième campagne (un an après la crue) ne concernent pas les stations 1 et 2 . Elles pourraient correspondre à un effet différé lié à l'augmentation du débit plancher: le peuplement de la station 3 se rapprocherait de celui de la station 2, tendant à la disparition des espèces dont l'abondance était liée au faible niveau du débit plancher. Nous ne disposons cependant pas d'un suivi suffisamment long pour confirmer cette hypothèse.

Les réponses des peuplements de poissons ne sont pas assez rapides pour dresser un bilan de l'effet de la crue d'une part, et de l'effet de l'augmentation du débit plancher d'autre part. Un tel bilan nécessiterait un suivi des peuplements sur plusieurs années, avec des difficultés d'interprétation dans le cas où d'autres événements hydrologiques sont susceptibles de se produire.

Les résultats que nous avons présentés permettent néanmoins de proposer un classement des modifications artificielles du débit en fonction du degré d'impact sur les peuplements de poissons : la situation la plus pénalisante est celle où le débit plancher est très faible, avec un peuplement différent des autres secteurs. Nous pouvons considérer que la crue entraîne des effets moins profonds que le régime d'éclusées avec débit plancher faible puisque la composition spécifique n'est pas modifiée après la crue. Le régime avec débit plancher soutenu correspondrait à la situation la moins pénalisante.

\subsection{Invertébrés benthiques et perturbations}

L'étude des invertébrés benthiques permet d'obtenir une réponse plus rapide. Ils ont un temps de récupération nette- 
ment plus faible que les poissons, du fait que leur cycle de vie est plus court (Niemi et al., 1990).

On constate que la crue centennale a eu un effet destructeur immédiat. La récupération est assez rapide après cet événement ponctuel, la diversité étant plus élevée dès le troisième mois suivant la crue. Ces mécanismes de réponse relèvent de l'élasticité du système traduisant sa capacité à revenir à l'état initial (Webster et al., 1983; Reice et al., 1990). On peut considérer que les peuplements ont retrouvé leur état initial lors de la cinquième campagne, réalisée six mois après la crue. Pour la station 3, il ne 's'agit cependant pas d'un état initial puisque le régime hydraulique a changé après la crue. Le peuplement se rapproche alors de celui de la station 2 soumise au même régime hydraulique; l'impact du régime à débit plancher faible semble donc réversible. L'abondance des taxons de milieu stagnant dans la station 3 avant relèvement du débit plancher s'explique par les faibles vitesses correspondantes, vitesses beaucoup plus variées et plus élevées à $1,35 \mathrm{~m}^{3} \cdot \mathrm{s}^{-1}$. Ce résultat permet de supposer l'existence de seuils de vitesses à débit plancher au-delà desquels les peuplements seraient moins déstructurés.

Les échantillonnages des peuplements d'invertébrés nous permettent de proposer un classement des situations observées. La situation avec débit plancher soutenu semble la moins pénalisante; la crue et le régime d'éclusées avec débit plancher faible sont responsables de bouleversements plus importants des peuplements. A partir des résultats, il est difficile de classer les deux dernières situations. Cependant, l'impact dans le temps est différent: la crue constitue un événement ponctuel, suivi d'une récupération des peuplements, alors que le régime d'éclusées est durable. Ce régime, lorsqu'il s'accompagne de déstructuration profonde, peut être considéré comme plus pénalisant que la crue centennale, car les effets persistent. Notons cependant que lorsque ce régime vient à changer, nous avons observé que la situation était réversible (station 3).

\subsection{Eléments d'aide à la gestion et au diagnostic}

Le classement proposé entre les deux régimes d'éćlusées de la Fontaulière permet d'apporter des éléments d'aide à la gestion des ouvrages hydroélectriques. II montre l'importance de définir un débit plancher soutenu (Valentin, 1995). S'il est trop faible, ce sont les conditions lentiques qui deviennent limitantes et qui entraînent les effets les plus marqués.

L'étude des deux secteurs de la Fontaulière ne nous permet pas de préconiser un seuil de débit plancher généralisable à partir d'un pourcentage du module $\left(1 / 9^{e}\right.$ et $1 / 100^{\circ}$ dans les deux stations étudiées) ou d'un rapport entre débit d'éclusée et débit plancher (respectivement 15 et 150). En revanche, cette étude nous a montré l'importance du maintien d'une diversité d'écoulement à débit plancher, avec des vitesses seuils dans les différents faciès des sites étudiés. A partir des résultats obtenus, on pourrait alors adopter une règle simple pour ce type de cours d'eau : maintenir dans les faciès lents des vitesses moyennes au moins égales à $8 \mathrm{~cm} \cdot \mathrm{s}^{-1}$ avec des vitesses locales atteignant 20 à $30 \mathrm{~cm} \cdot \mathrm{s}^{-1}$. Cette règle peut également constituer un élément de diagnostic si l'on veut évaluer le risque encouru par un système soumis à éclusées selon différents scénarios de débit plancher.

Dans le cas d'un diagnostic visant à évaluer l'effet d'un régime d'éclusées sur les peuplements aquatiques, nous pouvons suggérer quelques guides, à partir de l'étude de cas présentée ici : il apparaît en effet indispensable d'étudier la composition et la structure des peuplements, en comparaison à une situation de référence.

Ainsi, l'étude des peuplements de poissons ne devra pas s'appuyer uniquement sur la truite. Cette espèce n'est en effet pas la plus sensible aux variations de débit et ne peut pas, à elle seule, amener à conclure que le système ne subit pas d'effet des éclusées. De plus, les effectifs échantillonnés sont susceptibles d'être le reflet d'alevinages et ne permettent donc pas d'isoler l'effet de modifications hydrauliques.

La structure et la composition des peuplements d'invertébrés benthiques devra également être analysée en termes de diversité, richesse spécifique, densités et biomasses. Des indices globaux ne suffiront pas à évaluer les effets des modifications d'habitat sur les peuplements benthiques. De plus, l'étude de la cohérence entre la répartition des invertébrés en groupes trophiques et la biomasse algale permettra de juger du fonctionnement trophique du système.

L'hydroécologie propose donc désormais une quantification des altérations biologiques utilisable pour corriger des situations ou analyser l'effet de différentes options de gestion hydraulique des cours d'eau.

\section{Remerciements}

Ce travail de recherche été en partie financé par Electricité de France, Direction des Etudes et Recherches (contrat 2K1867). Il a pu être réalisé grâce à tous les membres du Laboratoire d'Hydroécologie Quantitative du Cemagref, et notamment à Pascal Roger par sa maîtrise du terrain. Yves Souchon, Marc Pouilly et Anne Thévenet ont été des relecteurs critiques et attentifs.

\section{Références}

[1] Carle F.L. \& Strub M.R. (1978). - A new method for estimating population size from removal data. Biometrics, $34,621-630$.

[2] Cowx I.G. (1983). - Review of the methods for estimating fish population size. from survey removal data. Fish management, 14(2), 67-82.

[3] Detenbeck N.E., Devore P.W., Niemi G.J. \& Lima A (1992). - Recovery of temperate-stream fish communities from disturbance : a rewiew of case studies and synthesis of theory. Environmental management, 16(1), 33-53.

[4] Gerdeaux D. (1987). - Revue des méthodes d'estimation de l'effectif d'une population par pêches successives avec retrait programme d'estimation d'effectif par la méthode de carle et strub. Bulletin Français de Pêche Piscicole, 304, 13-21.

[5] Horner R.R., Welch E.B. \& Veenstra R.B. (1983). Development of nuisance periphytic algae in laboratory streamsin relation to enrichment and velocity. In Wetzel R.G (Eds), Periphyton of Freshwater Ecosystems, Dr Junk W., The Hague, 121-134.

[6] Horton S. (1994). - Physical habitat use of two benthic species:-the bullhead (Cottus gobio L.) -the stone loach (Noemacheilus barbatulus L.). CEMAGREF, BEA/LHQ, technical report, $39 \mathrm{p}$.

[7] HyNES H.B.N. (1970). - The ecology of running waters. University of Toronto Press, Toronto. $555 \mathrm{p}$.

[8] LANCASTER J. \& HILDREW A. (1993). — Characterizing instream flow refugia. Canadian Journal of Fisheries and Aquatic Sciences, 50, 1663-1675.

[9] Malavoi J.R. (1989). — Typologie des faciès d'écoulement ou unités morphodynamiques des cours d'eau à haute énergie. Bulletin Français de la Pêche et de la Pisciculture, $315,189-210$ 
[10] Newbury R.W. (1984). - Hydrologic determinants of aquatic insect habitats. In Resh V.H. \& Rosenberg D.M. (Eds), The ecology of aquatic insects, Praeger, New York, 323-357.

[11] Niemi G.J., DeVore P., Detenbeck N., Taylor D., Lima A., PASTOR J., YOUNT J.D. \& R.J., N. (1990). - Overview of case studies on recovery of aquatic systems from disturbance. Environmental Management, 14, 571-587.

[12] POFF N.L. \& Ward J.V. (1989). - Implications of steamflow variability and predictability for lotic community structure : a regional analysis of streamflow patterns. Canadian Journal of Fisheries and Aquatic Sciences, 46, 1805 1818 .

[13] Power M.E., Stout R.J., Cushing C.E., Harper P.P., Hauer F.R., Matthews W.J., Moyle P.B., Statzner B. \& WAIS DE BADGEN I.R. (1988). - Biotic and abiotic controls in river and stream communities. Joumal of North American Benthological Society, 7, 456-479.

[14] Reice S.R., WiSSMaR R.C. \& Naiman R.J. (1990). - Disturbance regimes, resilience, and recovery of animal communities and habitats in lotic ecosystems. Environmental Management, 14, 647-659.

[15] Scarsbrook M.R. \& Townsend C.R. (1993). - Stream community structure in relation to spatial and temporal variation : a habitat templet study of two contrasting NewZealand streams. Freshwater Biology, 29, 395-410.

[16] Sedell R.J., Reeves G.H., Hauer F.R., Stanford J.A. \& HAwkINS C.P. (1990). - Role of refugia in recovery from disturbances : modern fragmented and disconnected river systems. Envirommental Management, 14, 711-724.

[17] Statzner B., Gore J.A. \& Resh V.H. (1988). - Hydraulic stream ecology: observed patterns and potential applications. Journal of the North American Benthological Society., 7(4), 307-360.

[18] Statzner B. \& Higler B. (1986). - Stream hydraulics as a major determinant of benthic invertebrate zonation patterns. Fres/water Biology, 16, 127-139.

[19] Valentin S. (1995). - Variabilité artificielle des conditions d'habitat et conséquences sur les peuplements aquatiques: effets écologiques des éclusées hvdroélectriques en rivière. Etudes de cas (Ance du Nord et Fontaulière) et approches expérimentales. Thèse de Doctorat, Lyon I, $284 \mathrm{p}$.

[20] Valentin S., Wasson J.G. \& Philippe M. (1995). Effects of hydropower peaking on epilithon and invertebrate community trophic structure. Regulated Rivers: Research \& Management, 10, 105-119.

[21] Webster J.R., Gurtz M.E., Hans J.J., Meyer J.L.. Swank W.T., Waide J.B. \& WALlace J.B. (1983). - Stability of stream ecosystems. in Barnes J.R. \& Minshall G.W. (Eds), Stream ecology: Application and testing of general ecological theory, Plenum Press, 355-395.

[22] Welch E.B., Jacoby J.M., Horner R.R. \& Seeley M.R. (1988). - Nuisance biomass levels of periphytic algae in streams. Hydrobiologia, 157, 161-168. 